\section{Histological changes of high axial myopia}

JB Jonas ${ }^{1}$ and $\mathrm{L} X \mathrm{u}^{2}$

\author{
Abstract \\ To describe pathological changes in the \\ anatomy of highly myopic (axially \\ elongated) eyes, enucleated globes were \\ examined by light microscopy and \\ ocular structures were measured \\ histomorphometrically. These studies \\ revealed that highly axially myopic eyes \\ show continuous thinning of the sclera \\ starting at or behind the equator with a \\ maximal thinning at the posterior pole; a \\ profound thinning of the choroid decreasing \\ from $\sim 250$ to $<10 \mu \mathrm{m}$ in extreme axial \\ myopia, secondary macular defects in the \\ Bruch's membrane associated with a \\ complete loss of retinal pigment epithelium \\ and choriocapillaris, and retinal \\ photoreceptors; a Bruch's membrane of \\ normal thickness in contrast to the profound \\ thinning of the choroid and the sclera; an up \\ to 10-fold elongation and thinning of the \\ peripapillary scleral flange as anterior roof \\ of the orbital cerebrospinal fluid space, and \\ subsequently a retrobulbar extension of the \\ cerebrospinal fluid space; an increased \\ distance of the peripapillary arterial circle \\ of Zinn-Haller to the optic disc border; \\ an elongation and thinning of the lamina \\ cribrosa with a subsequently decreased \\ distance between the intraocular pressure \\ compartment and the retrobulbar orbital \\ cerebrospinal fluid pressure compartment; \\ an increasing exposure of the peripheral \\ posterior lamina cribrosa surface to the \\ cerebrospinal fluid space, no longer \\ buffered by the solid optic nerve tissue; \\ and the development and enlargement of \\ parapapillary gamma zone, in contrast \\ to a myopia-independent parapapillary \\ beta zone. These anatomical changes \\ may be associated with high axial \\ myopia-related complications such as \\ an increased susceptibility of glauco- \\ matous optic neuropathy and myopic \\ retinopathy.
}

Eye (2014) 28, 113-117; doi:10.1038/eye.2013.223; published online 11 October 2013

Keywords: axial myopia; myopic retinopathy; Bruch's membrane; optic disc; peripapillary scleral flange; arterial circle Zinn-Haller

Axial myopia is defined as an axial length above the norm and too long for the refractive power of the whole optical system of the eye. Myopia, in particular high myopia, has become one of the major causes of visual field defects, visual impairment, and blindness. ${ }^{1,2}$ The importance of myopia will further increase because of the myopic shift taking place in particular in Asian countries at the Pacific rim. ${ }^{3-9}$ Recent population-based and community-based studies have shown that in schools in large Chinese cities, the prevalence of myopia (refractive error $\leq-1.00$ diopters) increased from $13 \%$ in the 7 -year-old to $46 \%$ in the 12 -year-old to $77 \%$ in the 18 -year-old teenagers. The prevalence of high axial myopia (refractive error $\leq-8.00$ diopters) was about $5 \% .{ }^{8,10} \mathrm{In}$ studies performed on university students in China in 2012, the prevalence of myopia increased to $95 \%$ and that of high myopia to 20\%. ${ }^{10}$ The increasing prevalence of myopia in children and young adults in China is likely to produce an associated increase in the development of potentially vision-threatening myopia-related complications in older adults. It may demonstrate the importance myopia may gain or already has gained.

\section{Definition of high axial myopia}

Previous population-based and hospital-based studies have revealed that axial myopia, in particular high axial myopia, is a risk factor for the development of rhegmatogenous retinal detachment, glaucomatous optic neuropathy, and myopic retinopathy, to name only a few. ${ }^{11,12}$

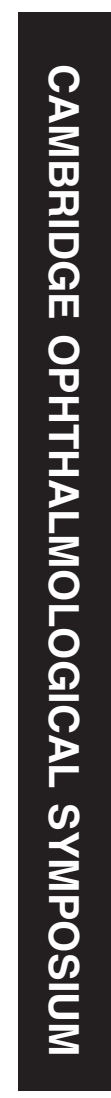

${ }^{1}$ Department of Ophthalmology, Medical Faculty Mannheim, Heidelberg University, Mannheim, Germany

${ }^{2}$ Beijing Institute of Ophthalmology, Beijing Tongren Hospital, Capital University of Medical Science, Beijing, China

Correspondence:

J B Jonas, Department of Ophthalmology, Medical

Faculty Mannheim, Heidelberg University, Theodor-Kutzer-Ufer 1-3, 68167 Mannheim,

Germany.

Tel: + 49-621-383-6078;

Fax: +49-621-383-3803.

E-mail: Jost.Jonas@

medma.uni-heidelberg.de

Received: 28 August 2013 Accepted in revised form: 5 September 2013 Published online: 11 October 2013 
The question arises what the cutoff value is to differentiate between moderate axial myopia and high axial myopia. If high axial myopia is characterized by an elongation of the globe, predominantly at the posterior pole, one may define the cutoff value for high axial myopia as the refractive error or axial length at which the size of the optic disc and the parapapillary atrophy markedly enlarges. Hospital-based and population-based investigations have revealed that the optic disc and the parapapillary atrophy start to enlarge at about a value of -8.00 diopters of refractive error or an axial length of $\sim 26.5 \mathrm{~mm} .{ }^{13-15}$ Beyond these values, the prevalence of myopic retinopathy and glaucomatous optic neuropathy steeply increased. ${ }^{11,12}$ One may therefore consider high axial myopia as a myopia refractive error of $>-8.00$ diopters or an axial length $>26.5 \mathrm{~mm}$.

\section{Histological changes in high axial myopia: sclera}

In non-axially elongated eyes with an axial length of $\leq 26.0 \mathrm{~mm}$, mean scleral thickness decreased from the limbus $(0.50 \pm 0.11 \mathrm{~mm})$ to the ora serrata $(0.43 \pm 0.14 \mathrm{~mm})$ and the equator $(0.42 \pm 0.15 \mathrm{~mm})$, and then increased to the midpoint between posterior pole and equator $(0.65 \pm 0.15 \mathrm{~mm})$ to the peri-optic nerve region at the merging point of the dura mater with the posterior sclera $(0.86 \pm 0.21 \mathrm{~mm})$, and finally to the posterior pole $(0.94 \pm 0.18 \mathrm{~mm}) .{ }^{16,17}$ The thickness of the peripapillary scleral flange as bridge between the lamina cribrosa of the optic nerve head and the posterior sclera was the lowest of all measurements $(0.39 \pm 0.09 \mathrm{~mm})$.

In highly axially myopic eyes, mean scleral thickness was significantly lower than in the non-highly myopic eyes for measurements taken at and posterior to the equator, whereas scleral thickness anterior to the equator did not differ significantly between highly axially myopic eyes and non-highly myopic eyes. ${ }^{16,17}$ It showed that the high axial myopia-associated thinning of the sclera was predominantly posterior to the equator and increased in proximity to the posterior pole of the eye. Correspondingly, scleral thickness measurements at or posterior to the equator were not significantly correlated with corneal thickness measurements, fitting with clinical studies in which central corneal thickness was not related with axial length. ${ }^{18}$ In the highly axially myopic group of globes, scleral thinning at the posterior pole and thinning of the peripapillary scleral flange were significantly correlated with thinning of the lamina cribrosa. Interestingly, globes with secondary high axial myopia due to congenital glaucoma showed a tendency to a thinning of the sclera in all regions including the region anterior to the cornea.

\section{Choroid/Bruch's membrane}

Clinical studies, population-based investigations and histological studies have shown a marked thinning of the choroid with increasing axial elongation. ${ }^{19}$ The mean choroidal thickness of $\sim 250 \mu \mathrm{m}$ in 65 -year-old emmetropic subjects decreased to $<30 \mu \mathrm{m}$ in highly axially myopic patients. It indicates that with increasing axial elongation, the distance between the Bruch's membrane and the sclera markedly decreased. The thickness of the Bruch's membrane, as examined in a recent histomorphometric study (own data), did not demonstrate a marked thinning in eyes with primary high axial myopia. Highly axially myopic eyes showed, however, defects in the Bruch's membrane in the macular region. ${ }^{20}$ These macular defects in the Bruch's membrane in highly axially myopic eyes may be called secondary defects as compared with the primary defect in the Bruch's membrane in the region of the optic nerve head. As the presence of the Bruch's membrane is essential for the presence of retinal pigment epithelium cells and choriocapillaris, the secondary macular defects in the Bruch's membrane were associated with a complete loss of retinal pigment epithelium cells and choriocapillaris, and an almost complete loss of the large choroidal vessel layer and photoreceptors. ${ }^{20}$ These macular Bruch's membrane defects can histologically be differentiated from myopic chorioretinal atrophic areas in the macular region with a complete loss of retinal pigment epithelium cells and choriocapillaris, but with the Bruch's membrane present, similar to the histology of geographic atrophy in the framework of age-related macular degeneration.

\section{Optic nerve head}

The optic nerve head can anatomically be regarded as a three-layered hole: a hole in the Bruch's membrane, a hole in the choroid flanked by the peripapillary border tissue, and a hole in the peripapillary scleral flange, porously covered by the lamina cribrosa. One may assume that at birth and in the early years of life, these three holes almost perfectly fit to each other. If axial myopia develops, the elongation of the globe takes place more in the posterior segment so that the position of the exit of the optic nerve (ie, the opening in the peripapillary scleral flange of the optic nerve head) moves from a location close to the posterior pole more to a location at the nasal wall of the globe. This shift in the position of the outer hole of the optic nerve head may not completely be followed by the opening in the Bruch's membrane, with the Bruch's membrane opening keeping a location closer to the posterior pole. It leads to an overhanging of the Bruch's membrane in the nasal region 
of the optic nerve head or optic disc (as also shown clinically by optical coherence tomography) and to an absence of the Bruch's membrane at the temporal disc border. ${ }^{21}$ The absence of the Bruchs membrane in the parapapillary region has recently been examined and measured in histomorphometric studies. The region without the Bruch's membrane (and correspondingly, without retinal pigment epithelium and choriocapillaris) has been called parapapillary gamma zone. ${ }^{20,22,23}$ This gamma zone was significantly correlated with high axial myopia, with the prevalence of gamma zone steeply increasing at a cutoff value of $\sim 26.0 \mathrm{~mm}$ of axial length (in histologically fixed globes). Interestingly,

parapapillary gamma zone was not related with the presence of glaucomatous optic nerve damage.

A parapapillary beta zone histologically defined by the presence of the Bruch's membrane and absence of the retinal pigment epithelium (associated with an almost complete loss of retinal photoreceptors and an almost complete closure of choriocapillaris) was not associated with axial length, but with the presence of glaucoma. ${ }^{22,23}$ These histomorphometric studies were confirmed by clinical studies applying the enhanced depth imaging mode of optical coherence tomography to image and measure the parapapillary region. ${ }^{23}$ The optic nerve head (defined ophthalmoscopically as the opening in the peripapillary scleral flange, also called 'scleral canal') shows a marked enlargement with increasing axial length starting at an axial length of $\sim 26.5 \mathrm{~mm}$ or a myopic refractive error of about -8.00 diopters. ${ }^{13-15}$ Although in non-highly myopic eyes, the optic disc size is mostly independent or only slightly dependent on axial length, highly axially myopic eyes show a relatively strong association between axial length and optic disc size. ${ }^{24,25}$ The enlarged optic disc in highly axially myopic eyes as so-called 'secondary or acquired macrodiscs' can thus be differentiated from 'primary macrodiscs' in nonhighly myopic eyes. The size of primary macrodiscs is not related with axial length nor with age, whereas it is related to the number of retinal pigment epithelium cells, photoreceptors, retinal ganglion cell axons, and number of total size of lamina cribrosa pores. ${ }^{26-28}$ In contrast, the size of secondary macrodiscs is related to axial length and age, and may not be related to the number of pigment epithelium cells, photoreceptors, and retinal ganglion cell axons.

Parallel to the high axial myopia associated enlargement of the optic nerve head, the lamina cribrosa as the porous cover plate of the opening in the peripapillary scleral flange shows a stretching and thinning. ${ }^{29,30}$ It decreases the distance between the intraocular pressure compartment and the retrolaminar orbital cerebrospinal fluid pressure compartment. ${ }^{31}$ With the pressures in both compartments unchanged (ie, an unchanged trans-lamina cribrosa pressure difference), a reduced distance leads to an steepened pressure gradient between both compartments. This may be one of the reasons (besides morphological changes in the stretched and elongated lamina cribrosa, and thinning of the peripapillary scleral flange) that is responsible for the increased glaucoma susceptibility of highly myopic eyes. ${ }^{11}$

With the increase in the aperture of the peripapillary scleral flange opening (so-called scleral canal of the optic nerve head) but with the optic nerve remaining constant in size, the peripheral posterior surface of the lamina cribrosa is no longer covered and buffered by the solid tissue of the optic nerve head, but gets exposed to the orbital cerebrospinal fluid space. ${ }^{30}$ As the fluid offers much less mechanical resistance against a deformation than the solid optic nerve tissue, pressure-related pathological changes may predominantly take place in the periphery of the lamina cribrosa close at the optic disc border.

This may be the anatomic basis for the development of so-called acquired pits of the optic nerve head ('APONs') in glaucomatous eyes. ${ }^{32,33}$ These APONS are typically located sharply at the optic disc border at the 6 o'clock position or 12 o'clock position. They are located at the disc border, as the peripheral outer surface of the lamina cribrosa may no longer be supported by the optic nerve tissue. They are located at the vertical disc poles, as the optic disc shape is usually slightly vertically oval, so that, with a circular optic nerve shape, the vertical poles are the first regions where the posterior lamina cribrosa surface gets exposed to the cerebrospinal fluid space. ${ }^{30}$ Interestingly, clinical studies have shown that the early glaucomatous visual field defects and early retinal nerve fiber layer loss correspond to a loss of retinal ganglion cells located close to the temporal raphe, with the axons originating in that area being located close at the optic disc border within the optic nerve head. ${ }^{34}$

In the parapapillary region, highly axially myopic eyes show, in addition to the parapapillary gamma zone, a marked elongation and corresponding thinning of the peripapillary scleral flange. ${ }^{35}$ If the normal thickness of the flange is about $0.40 \mathrm{~mm}$, it can get reduced to $50 \mu \mathrm{m}$ in highly axially myopic eyes, parallel to an elongation of about $0.5-5.0 \mathrm{~mm}$. As the peripapillary scleral flange is the biomechanical anchor of the lamina cribrosa, any weakening of the flange, such as thinning, may have a consequence for the biomechanical stability of the lamina cribrosa and the fibers passing through it. ${ }^{36}$

The peripapillary border tissue ('of Elschnig') is in its thickness mostly independent of the axial length of the globe (own data). The so-called peripapillary ring is probably just the peripapillary border tissue that is the continuation of the pia mater of the optic nerve. In highly 
axially myopic eyes with a large parapapillary gamma zone (ie, with the Bruchs membrane absent at the optic disc border), the ophthalmoscopically detectable peripapillary ring may be just the pia mater of the optic nerve.

As the peripapillary arterial circle of Zinn-Haller is located approximately at the merging point of the dura mater with the posterior sclera (ie, the starting point of the peripapillary scleral flange), ${ }^{37}$ the high axial myopiaassociated elongation of the flange leads to an increased distance between the arterial circle of Zinn-Haller and the optic disc border (own data). ${ }^{38}$ As the arterial circle is the main arterial source for the lamina cribrosa blood supply, ${ }^{39,40}$ it has been discussed whether the high axial myopia induced increased distance between the arterial circle, and the lamina cribrosa may be of importance for the pathogenesis of an increased glaucoma susceptibility in highly myopic eyes.

These anatomical findings and facts may give space to speculate upon, such as whether one should consider pathological progressive myopia as a special type of normal-pressure glaucoma. This may be justified as high axial myopia is a risk factor for glaucomatous optic neuropathy even in eyes with a normal intraocular pressure. Highly axial myopic glaucoma may be, however, only a special subtype of 'normal-pressure glaucoma', as in the highly axially myopic eyes anatomical peculiarities of the optic nerve head may be the reason for the increased glaucoma susceptibility. It may be in contrast to non-highly myopic normalpressure glaucoma for which an abnormally low orbital cerebrospinal fluid pressure among other actors has been discussed to be pathogenetically important. ${ }^{41,42}$ Another question is whether one can use traditional functional clinical tests (eg, visual field, disc (or posterior pole) photos, or macular and optic nerve head optical coherent tomographic images) for differentiating pathologically high myopia-related visual loss from visual loss due to glaucomatous optic neuropathy. Depending on the clinical situation, the most sensitive technique may be the ophthalmoscopical examination of the optic nerve head and searching for fine but definite kinking of retinal blood vessels close to the optic disc border. This may indicate a glaucomatous loss of neuroretinal rim, almost pathognomonic for glaucoma. It may be relatively difficult to detect this slight vessel kinking with the modern imaging techniques. Another question could be whether lowering of intraocular pressure is therapeutically helpful for the therapy of high axial myopia-related glaucomatous optic neuropathy. Although there may not be studies definitely answering this question, one may assume that lowering of the translamina cribrosa pressure difference may be helpful to support stressed retinal ganglion cell axons within the lamina cribrosa. It has not been intensively examined whether lowering of intraocular pressure is helpful to prevent high axial myopia-related changed in the macular region.

In conclusion, high axial myopia is associated with numerous anatomical changes in the posterior pole of the globe. These changes that can also be imaged by optical coherence tomography may explain some of the associations between high myopia and visionthreatening diseases such as glaucoma and myopic retinopathy.

\section{Conflict of interest}

The authors declare no conflict of interest.

\section{References}

1 Xu L, Wang Y, Li Y, Li J, Wang Y, Cui T et al. Causes of blindness and visual impairment in an urban and rural area in Beijing: the Beijing Eye Study. Ophthalmology 2006; 113(1141): e1-e3.

2 Wang $\mathrm{Y}, \mathrm{Xu} \mathrm{L}$, Jonas JB. Prevalence and causes of visual field loss as determined by frequency doubling perimetry in urban and rural adult Chinese. Am J Ophthalmol 2006; 141: 1078-1086.

3 Zhao J, Pan X, Sui R, Munoz SR, Sperduto RD, Ellwein LB. Refractive error study in children: results from Shunyi District, China. Am J Ophthalmol 2000; 129: 427-435.

4 He M, Zeng J, Liu Y, Xu J, Pokharel GP, Ellwein LB. Refractive error and visual impairment in urban children in southern China. Invest Ophthalmol Vis Sci 2004; 45: 793-799.

5 Congdon N, Wang Y, Song Y, Choi K, Zhang M, Zhou Z et al. Visual disability, visual function, and myopia among rural Chinese secondary school children: the Xichang Pediatric Refractive Error Study (X-PRES)-report 1. Invest Ophthalmol Vis Sci 2008; 49: 2888-2894.

6 O'Donoghue L, McClelland JF, Logan NS, Rudnicka AR, Owen CG, Saunders KJ. Refractive error and visual impairment in school children in Northern Ireland. Br J Ophthalmol 2010; 94: 1155-1159.

7 Guo Y, Liu LJ, Xu L, Lv YY, Tang P, Feng Y et al. Outdoor activity and myopia among primary students in rural and urban regions in Beijing. Ophthalmology 2013; 120: 277-283.

8 Morgan IG, Ohno-Matsui K, Saw SM. Myopia. Lancet 2012; 379: 1739-1748.

9 Sun J, Zhou J, Zhao P, Lian J, Zhu H, Zhou Y et al. High prevalence of myopia and high myopia in 5060 Chinese university students in Shanghai. Invest Ophthalmol Vis Sci 2012; 53: 7504-7759.

10 Xu L, Wang Y, Wang S, Wang Y, Jonas JB. High myopia and glaucoma susceptibility. The Beijing Eye Study. Ophthalmology 2007; 114: 216-220.

11 Liu HH, Xu L, Wang YX, Wang S, You QS, Jonas JB. Prevalence and progression of myopic retinopathy in Chinese adults: The Beijing Eye Study. Ophthalmology 2010; 117: $1763-1768$

12 Jonas JB. Optic disc size correlated with refractive error. Am J Ophthalmol 2005; 139: 346-348.

13 Wang $Y, X u$ L, Zhang L, Yang H, Ma Y, Jonas JB. Optic disk size in a population-based study in Northern China. The Beijing Eye Study. Br J Ophthalmol 2006; 90: 353-356. 
14 Xu L, Wang YX, Wang S, Xu L, Wang YX, Wang S et al. Definition of high myopia by parapapillary atrophy. The Beijing Eye Study. Acta Ohthalmol 2010; 88: e350-e351.

15 Heine L. Beiträge zur Anatomie des myopischen Auges. Arch Augenheilk 1899; 38: 277-290.

16 Vurgese S, Panda-Jonas S, Jonas JB. Sclera thickness in human globes and its relations to age, axial length and glaucoma. PLoS One 2012; 7: e29692.

17 Nangia V, Jonas JB, Sinha A, Matin A, Kulkarni M. Central corneal thickness and its association with ocular and general parameters in Indians: The Central India Eye and Medical Study. Ophthalmology 2010; 117: 705-710.

18 Wei WB, Xu L, Jonas JB, Shao L, Du KF, Wang S et al. Subfoveal choroidal thickness: the Beijing Eye Study. Ophthalmology 2013; 120: 175-180.

19 Jonas JB, Ohno-Matsui K, Spaide RF, Holbach L, Panda-Jonas S. Macular Bruchs membrane holes in high myopia: Associated with gamma zone and delta zone of parapapillary region. Invest Ophthalmol Vis Sci 2013; 54: 1295-1230.

20 Reis AS, Sharpe GP, Yang H, Nicolela MT, Burgoyne CF, Chauhan BC. Optic disc margin anatomy in patients with glaucoma and normal controls with spectral domain optical coherence tomography. Ophthalmology 2012; 119: 738-747.

21 Jonas JB, Jonas SB, Jonas RA, Holbach L, Dai Y, Sun X et al. Parapapillary atrophy: Histological gamma zone and delta zone. PLoS One 2012; 7: e47237.

22 Dai Y, Jonas JB, Huang H, Wang M, Sun X. Microstructure of parapapillary atrophy: Beta zone and gamma zone. Invest Ophthalmol Vis Sci 2013; 54(3): 2013-2018.

23 Jonas JB, Gusek GC. Naumann GOH. Optic disk morphometry in high myopia. Graefes Arch Clin Exp Ophthalmol 1988; 226: 587-590.

24 Xu L, Li Y, Wang S, Wang Y, Wang Y, Jonas JB Characteristics of highly myopic eyes. The Beijing Eye Study. Ophthalmology 2007; 114: 121-126.

25 Panda-Jonas S, Jonas JB, Jakobczyk M, Schneider U. Retinal photoreceptor count, retinal surface area, and optic disc size in normal human eyes. Ophthalmology 1994; 101: 519-523.

26 Panda-Jonas S, Jonas JB, Jakobczyk-Zmija M. Retinal pigment epithelium cell count, density and correlations in normal human eyes. Am J Ophthalmol 1996; 121: 181-189.

27 Jonas JB, Schmidt AM, Müller-Bergh JA, SchlötzerSchrehardt UM, Naumann GO. Human optic nerve fiber count and optic disc size. Invest Ophthalmol Vis Sci 1992; 33 2012-2018.

28 Dichtl A, Jonas JB. Naumann GOH. Histomorphometry of the optic disc in highly myopic eyes with glaucoma. $\mathrm{Br} J$ Ophthalmol 1998; 82: 286-289.
29 Jonas JB, Berenshtein E, Holbach L. Lamina cribrosa thickness and spatial relationships between intraocular space and cerebrospinal fluid space in highly myopic eyes. Invest Ophthalmol Vis Sci 2004; 45: 2660-2665.

30 Jonas JB, Berenshtein E, Holbach L. Anatomic relationship between lamina cribrosa, intraocular space, and cerebrospinal fluid space. Invest Ophthalmol Vis Sci 2003; 44: 5189-5195.

31 Javitt JC, Spaeth GL, Katz LJ, Poryzees E, Addiego R. Acquired pits of the optic nerve. Increased prevalence in patients with low-tension glaucoma. Ophthalmology 1990; 97: 1038-1043.

32 You JY, Park SC, Su D, Teng CC, Liebmann JM, Ritch R. Focal lamina cribrosa defects associated with glaucomatous rim thinning and acquired pits. JAMA Ophthalmol 2013; 131: 314-320.

33 Gramer E, Gerlach R, Krieglstein GK, Leydhecker W. [Topography of early glaucomatous visual field defects in computerized perimetry]. Klin Monbl Augenheilkd 1982; 180: 515-523.

34 Jonas JB, Jonas SB, Jonas RA, Holbach L, Panda-Jonas S Histology of the parapapillary region in high myopia. Am J Ophthalmol 2011; 152: 1021-1029.

35 Burgoyne CF, Morrison JC. The anatomy and pathophysiology of the optic nerve head in glaucoma. J Glaucoma 2001; 10(5 Suppl 1): S16-S18.

36 Jonas JB, Jonas SB. Histomorphometry of the circular peripapillary arterial ring of Zinn-Haller in normal eyes and eyes with secondary angle-closure glaucoma. Acta Ophthalmol 2010; 88: e317-e322.

37 Ohno-Matsui K, Kasahara K, Moriyama M. Detection of Zinn-Haller arterial ring in highly myopic eyes by simultaneous indocyanine green angiography and optical coherence tomography. Am J Ophthalmol 2013; 155: 920-926.

38 Hayreh SS. Blood supply of the optic nerve head and its role in optic atrophy, glaucoma, and oedema of the optic nerve. Br J Ophthalmol 1969; 53: 721-748.

39 Hayreh SS. Anatomy and physiology of the optic nerve head. Trans Am Acad Ophthalmol Otolaryngol 1974; 78: 240-254.

40 Ren R, Jonas JB, Tian G, Zhen Y, Ma K, Li S et al. Cerebrospinal fluid pressure in glaucoma. A prospective study. Ophthalmology 2010; 117: 259-266.

41 Berdahl JP, Fautsch MP, Stinnett SS, Allingham RR. Intracranial pressure in primary open angle glaucoma, normal tension glaucoma, and ocular hypertension: a casecontrol study. Invest Ophthalmol Vis Sci 2008; 49: 5412-5418. 